\title{
The Dynamics of Abnormal Grain Growth in a Particle-Containing System: Integration of 3D Experimental Data into a Capillarity Driven Model
}

\author{
Ning $\mathrm{Lu}^{1 *}$, Jiwoong Kang ${ }^{1}$, Nancy Senabulya ${ }^{2}$, Nicolas Gueninchault ${ }^{3}$, Ashwin J. Shahani ${ }^{*}$ \\ 1. Department of Materials Science and Engineering, University of Michigan, Ann Arbor, MI, USA. \\ 2. Michigan Center for Materials Characterization, University of Michigan, Ann Arbor, MI, USA. \\ 3. Xnovo Technology ApS, Køge, Denmark. \\ * Corresponding author: Ning Lu: lning@umich.edu, Ashwin J. Shahani: shahani@umich.edu
}

Grain growth is a common phenomenon that occurs during materials processing. In most cases, the grain size follows a normal distribution after deformation and recrystallization [1]. The corresponding probability distribution smoothly shifts to larger grain sizes as annealing proceeds; this process is referred to as normal grain growth. However, in some cases, a few grains "run away" from the normal distribution and grow significantly faster than the other grains. Those grains become abnormally large and the ensuing process is known as abnormal grain growth (AGG).

In a particle-containing system, the grain growth rate is determined by the competition between a driving force for grain growth and a retarding Zener pinning force induced by the particle cloud. This pinning force can be estimated from the equilibrium condition, wherein GBs motion is completely pinned [2]. Thus, the question remains on how to evaluate the driving force. Andersen et al. [3] proposed a 2D geometric model in which an abnormal grain is surrounded by equivalent grains and from this calculated the interface curvature. Razzak et al. [4] proposed a similar 3D analytic model to estimate the driving force by treating it as the reduction of GB energy. Based on their approach, in the equilibrium condition, it was found that the grains with a size greater than about 1.4 times the mean grain size will grow abnormally. However, those models were not cross-checked against experimental results, likely due to the dearth of in situ and 3D metrologies. Recently, a capillarity driven model for grain growth was extended from 2D [5] to 3D [6]. Critical geometric parameters for normal grain growth were successfully predicted and explained by 3D capillarity driven model [7]. Therefore, it is desirable to apply this model to investigate AGG.

Here, an Al-3.5 wt.\% Cu alloy was used to investigate AGG [1]. X-ray diffraction contrast tomography (DCT) and absorption contrast tomography (ACT) were conducted on a laboratory X-ray microscope (Zeiss Xradia 520 Versa) at the University of Michigan. Initially, the sample was annealed at $400{ }^{\circ} \mathrm{C}$ for $30 \mathrm{~min}$. after cold rolling followed by homogenization at $550{ }^{\circ} \mathrm{C}$ for $4 \mathrm{hrs}$. Subsequently, AGG was observed after cumulative annealing at $485{ }^{\circ} \mathrm{C}$ for $97 \mathrm{~min}$., as indicated by a large grain (blue) in Fig. 1(a). The measured grain size of 99 normal grains $\left(R_{n}\right)$ and a single abnormal grain $\left(R_{a b}\right)$ are 32-253 and 394 $\mu \mathrm{m}$, respectively. The mean grain size $\left(R_{n}^{m}\right)$ of normal grains is $142 \mu \mathrm{m}$. The particle distribution of $\theta$ $\mathrm{Al}_{2} \mathrm{Cu}$ particles is shown in Fig. 1(b,c). Clearly, the distribution of particles is non-uniform. One reason for this is that homogenization treatment is insufficient and therefore there is still a segregation of $\mathrm{Cu}$ in the sample.

Combining capillarity (in 3D) with Zener pinning, grain growth rate was determined as

$$
\frac{d R_{a b}}{d t}=M \gamma\left(\frac{1}{R_{n}^{m}}-\frac{1}{R_{a b}}-\frac{1}{2 R_{0}}\right)
$$




$$
\frac{d R_{n}^{m}}{d t}=M \gamma\left(\frac{1}{2 R_{n}^{m}}-\frac{1}{2 R_{0}}\right)
$$

where $M$ is the GB mobility, $\gamma$ is GB energy and $R_{0}$ is the limited grain size when GBs motion is pinned by particles. In our case it can be assumed that mean $R_{n}^{m}$ equals to $R_{0}$, as most of the normal grains do not continue growing. In order for AGG to take place, both the absolute and relative growth rate should be greater than zero, i.e., $\frac{d R_{a b}}{d t}>0$ and $\frac{d}{d t}\left(\frac{R_{a b}}{R_{n}^{m}}\right)>0$. Under these conditions, and invoking the previous two models [3-4] as well as our own (Eqs. (1-2)), a "mechanism map" can be constructed, see Fig. 2. In order to better understand AGG in our case, the grain size ratio $\left(R / R_{n}^{m}\right.$, including $R_{a b} / R_{n}^{m}$ and $\left.R_{n} / R_{n}^{m}\right)$ at $t=97 \mathrm{~min}$ is also plotted in Fig. 2. $R_{a b} / R_{n}^{m}$ is marked by red color and the distribution of $R_{n} / R_{n}^{m}$ (representing the probability density of grain size $R_{n}$ ), is presented by a symmetric blue outline. That is, the width of this outline along the $R_{n}^{m} / R_{0}$ axis indicates probability density of corresponding $R_{n}$. The mechanism map derived using the capillarity driven model reveals that AGG will be triggered when $R_{a b} / R_{n}^{m}>2$, which is a little higher than in previous models. Nevertheless, it is consistent with our 3D observation that $R_{n} / R_{n}^{m}$ (blue outline) are all below two, but $R_{a b} / R_{n}^{m}$ (red point) is greater than this critical value.

\section{References:}

[1] J Dennis et al., Acta Mater. 57 (2009), p. 4539.

[2] CS Smith, Trans. AIME, 175 (1948), p. 15.

[3] I Andersen et al., Acta Metall. Mater., 43 (1995), p. 2689.

[4] M Razzak et al., ISIJ Int., 52 (2012), p. 2278.

[5] JV Neumann in "Metal Interfaces", ed. C. Herring, (American Society for Metals, Cleveland) p. 108.

[6] R MacPherson et al., Nature 446 (2007), p. 1053.

[7] D Rowenhorst et al., Acta Mater., 58 (2010), p. 5511.
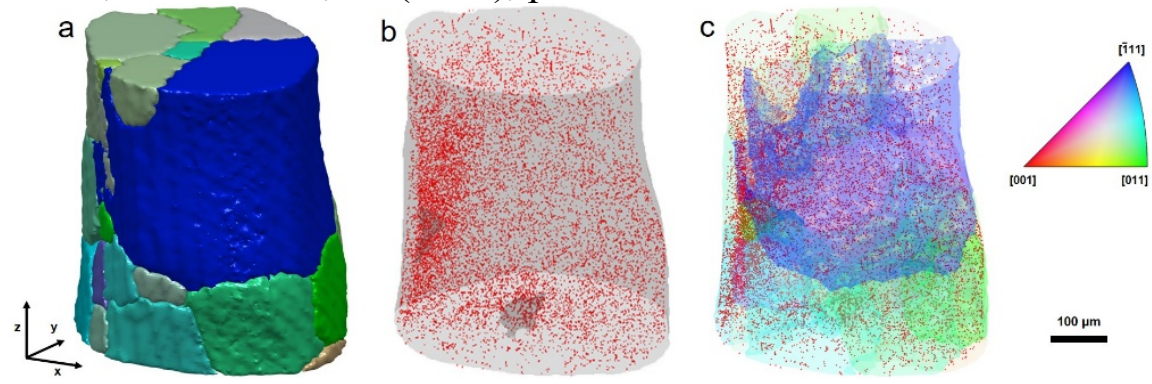

Figure 1. (a) 3D grain map of $\mathrm{Al}-3.5 \mathrm{wt} \% \mathrm{Cu}$ sample, obtained from DCT. (b) $3 \mathrm{D}$ distribution of $\theta-\mathrm{Al}_{2} \mathrm{Cu}$ particles, from ACT. (c) Superposition of grain map and particle locations.

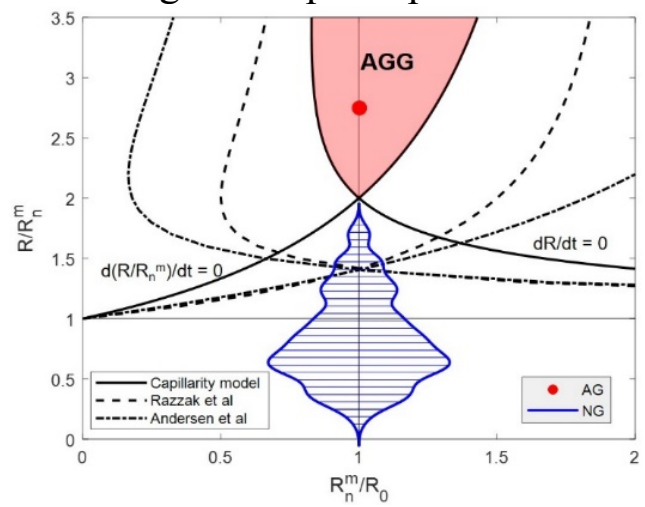

Figure 2. Mechanism map for AGG based on three models. 